\title{
RAMANUJAN'S ASSOCIATION WITH RADICALS IN INDIA
}

\author{
Bruce C. Berndt, Heng Huat Chan, and Liang-Cheng Zhang \\ In memory of Ramanujan on the
$\left(32\left(\frac{146410001}{48400}\right)^{3}-6\left(\frac{146410001}{48400}\right)+\sqrt{\left(32\left(\frac{146410001}{48400}\right)^{3}-6\left(\frac{146410001}{48400}\right)\right)^{2}-1}\right)^{1 / 6} t h$
anniversary of his birth
}

Ramanujan (sometimes under the alternative spelling Ramanujam) submitted 58 problems to the Journal of the Indian Mathematical Society. Approximately ten of them involve equalities between radicals. For example [16, p. 334],

$$
\left(\sqrt[5]{\frac{32}{5}}-\sqrt[5]{\frac{27}{5}}\right)^{1 / 3}=\sqrt[5]{\frac{1}{25}}+\sqrt[5]{\frac{3}{25}}-\sqrt[5]{\frac{9}{25}}
$$

Establishing equalities among exotic radicals was very common in Ramanujan's day, especially in Great Britain and its empire. For example, see the then popular texts by H. S. Hall and S. R. Knight [12, Chap. 8] and G. Chrystal [10, Chap. 11], the latter being well-known to Ramanujan. Was Ramanujan's keen interest in radical equalities merely a consequence of their popularity in his time, or were there other reasons? The answer can be found in his notebooks [15] and in one of his most important papers [14], [16, pp. 23-39].

Scattered among the pages in Ramanujan's first notebook are the values of 107 class invariants, or polynomials satisfied by them. As we shall see, these invariants frequently take the shapes of interesting radicals, and often to put the radical expressions in their most attractive forms, difficult radical equalities need to be established. So that we may define Ramanujan's class invariants, set

$$
\chi(q)=\prod_{n=0}^{\infty}\left(1+q^{2 n+1}\right)
$$

For any positive rational number $n$, set

$$
q=\exp (-\pi \sqrt{n})
$$

and define the two class invariants $G_{n}$ and $g_{n}$ by

$$
G_{n}:=2^{-1 / 4} q^{-1 / 24} \chi(q) \quad \text { and } \quad g_{n}:=2^{-1 / 4} q^{-1 / 24} \chi(-q) \text {. }
$$

As we shall see, we are able to calculate $G_{n}$ for certain odd values of $n$ and $g_{n}$ for certain even values of $n$. 
At the beginning of the twentieth century, these invariants were extensively studied by H. Weber [21], who used the notations $G_{n}=: 2^{-1 / 4} \mathfrak{f}(\sqrt{-n})$ and $g_{n}=$ : $2^{-1 / 4} \mathfrak{f}_{1}(\sqrt{-n})$. Weber [21] proved that $G_{n}$ and $g_{n}$ are algebraic. In fact, $G_{n}$, $2^{-1 / 12} G_{n}$, and $2^{-1 / 4} G_{n}$ are units in some algebraic number field according as $n \equiv 1$ $(\bmod 4), n \equiv 3(\bmod 8)$, and $n \equiv 7(\bmod 8)$, respectively. If $n \equiv 2(\bmod 4)$, then $g_{n}$ is a unit. Weber's study of $G_{n}$ and $g_{n}$ was motivated by the construction of the Hilbert class field $H_{n}$, the maximal unramified abelian extension of the imaginary quadratic field $K_{n}:=\mathbb{Q}(\sqrt{-n})$. It can be shown that $H_{n}=K_{n}\left(j\left(\omega_{n}\right)\right)$, where

$$
\omega_{n}= \begin{cases}\sqrt{-n}, & \text { if } n \equiv 1(\bmod 4), \\ \frac{3+\sqrt{-n}}{2}, & \text { if } n \equiv 3(\bmod 4),\end{cases}
$$

and $j$ is the famous modular $j$-invariant, so-called because it is invariant under transformations from the modular group. Weber [21] asserted that certain small powers of $G_{n}$ and $g_{n}$ can be used to replace $j\left(\omega_{n}\right)$ as generators of $H_{n}$ over $K_{n}$. Perhaps for these reasons, Weber called $G_{n}$ and $g_{n}$ class invariants, and computed a total of 105 class invariants or the monic, irreducible polynomials satisfied by them. The excellent text of D. A. Cox [11] provides an accessible account of Weber's work on invariants.

Before proceeding further, we give some examples that Ramanujan calculated:

$$
\begin{gathered}
G_{5}=\left(\frac{1+\sqrt{5}}{2}\right)^{1 / 4} \\
G_{17}=\sqrt{\frac{5+\sqrt{17}}{8}}+\sqrt{\frac{\sqrt{17}-3}{8}} \\
G_{69}=\left(\frac{5+\sqrt{23}}{\sqrt{2}}\right)^{1 / 12}\left(\frac{3 \sqrt{3}+\sqrt{23}}{2}\right)^{1 / 8}\left(\sqrt{\frac{6+3 \sqrt{3}}{4}}+\sqrt{\frac{2+3 \sqrt{3}}{4}}\right)^{1 / 2}
\end{gathered}
$$

and

$$
\begin{aligned}
G_{1353}= & \left(\frac{3+\sqrt{11}}{\sqrt{2}}\right)^{1 / 4}\left(\frac{5+3 \sqrt{3}}{\sqrt{2}}\right)^{1 / 4}\left(\frac{11+\sqrt{123}}{\sqrt{2}}\right)^{1 / 4}\left(\frac{6817+321 \sqrt{451}}{\sqrt{2}}\right)^{1 / 12} \\
& \times\left(\sqrt{\frac{17+3 \sqrt{33}}{8}}+\sqrt{\frac{25+3 \sqrt{33}}{8}}\right)^{1 / 2} \\
& \times\left(\sqrt{\frac{561+99 \sqrt{33}}{8}}+\sqrt{\frac{569+99 \sqrt{33}}{8}}\right)^{1 / 2} .
\end{aligned}
$$

The value of $G_{69}$ was only recently verified for the first time by the authors [7]. In our calculation of $G_{69}$, we used the equality

$$
\left(188+108 \sqrt{3}+\sqrt{(188+108 \sqrt{3})^{2}-1}\right)^{1 / 6}=\sqrt{\frac{6+3 \sqrt{3}}{4}}+\sqrt{\frac{2+3 \sqrt{3}}{4}}
$$


which is the special case, $a=(4+3 \sqrt{3}) / 4$, of the more general equality

$$
\left(32 a^{3}-6 a+\sqrt{\left(32 a^{3}-6 a\right)^{2}-1}\right)^{1 / 6}=\sqrt{a+\frac{1}{2}}+\sqrt{a-\frac{1}{2}},
$$

which we also used in the dedication at the beginning of this paper and in calculations of further class invariants.

The value of $G_{1353}$ was communicated by Ramanujan [16, p. xxix, eq. (23)], [8, p. 62] in his second letter, dated 27 February 1913, to G. H. Hardy and was first established (unrigorously) by G. N. Watson [18]. In a letter of 1 October 1930 to B. M. Wilson [8, pp. 237, 238], Watson confided, " ... but 23 which deals with the singular modulus associated with 1353 is included; I was pleased at getting this out, because the bulk of the singular moduli in the Notebooks can be obtained in the same way ... You will be interested to hear how Ramanujan got no. 23, particularly when you look at the length of the answer. I am absolutely convinced that he guessed it." (Calculating a singular modulus, which we do not define here, is equivalent to calculating a class invariant.) The reader is undoubtedly astonished to learn that Ramanujan first "guessed" his formula for $G_{1353}$. We do not agree with Watson! We think that Watson's proof, which is not rigorous, could not have been given without his knowing the formula in advance. The first rigorous proof was given recently by Chan [9].

On pages 294-299 in his second notebook [15], Ramanujan gave a table of values for 77 class invariants, three of which are not found in the first notebook. Since the second notebook is an enlarged revision of the first, it is unclear why Ramanujan failed to record 33 class invariants that he offered in the first notebook. Four further results are found in scattered places in the second notebook. After arriving in Cambridge, Ramanujan learned of Weber's work [21], and so when he wrote his paper [14], [16, pp. 23-39], the table of 46 class invariants that he included did not contain any that are found in Weber's book [21]. Except for $G_{325}$ and $G_{363}$, all of the remaining values are found in Ramanujan's notebooks. To the best of our reckoning, Ramanujan calculated a total of 116 class invariants, or monic, irreducible polynomials satisfied by them.

Why did Ramanujan calculate such a large number of class invariants? Ramanujan did not share Weber's interest in generating Hilbert class fields, but he did have applications. First, as the title of his paper [14] indicates, Ramanujan used class invariants to find excellent approximations to $\pi$. For example, from (1) and (3), we find that

$$
\pi \approx \frac{24}{\sqrt{69}}\left(\log G_{69}+\frac{1}{4} \log 2\right)=3.1415926536032 \ldots,
$$

which agrees with the value of $\pi$ through nine decimal places.

Second, Ramanujan used class invariants to determine explicitly particular values of the theta function $\varphi(q)$ defined by

$$
\varphi(q):=\sum_{k=-\infty}^{\infty} q^{k^{2}}
$$

For example, Ramanujan probably used his value of $G_{49}$ to show that [3]

$$
\frac{\varphi^{2}\left(e^{-7 \pi}\right)}{\varphi^{2}\left(e^{-\pi}\right)}=\frac{\sqrt{13+\sqrt{7}}+\sqrt{7+3 \sqrt{7}}}{14}(28)^{1 / 8} .
$$


The value

$$
\varphi\left(e^{-\pi}\right)=\frac{\pi^{1 / 4}}{\Gamma\left(\frac{3}{4}\right)}
$$

is well known [1, p. 103], and so (4) provides an explicit evaluation for $\varphi\left(e^{-7 \pi}\right)$.

The theta function $\varphi$ is intimately connected with elliptic functions and integrals. An elliptic function is a function of a complex variable with two linearly independent periods, in contrast to the familiar trigonometric functions which have just one linearly independent period. The complete elliptic integral of the first kind associated with the modulus $k, 0<k<1$, is defined by

$$
K:=K(k):=\int_{0}^{\pi / 2} \frac{d \theta}{\sqrt{1-k^{2} \sin ^{2} \theta}} .
$$

The complementary modulus $k^{\prime}$ is defined by $k^{\prime}=\sqrt{1-k^{2}}$; set $K^{\prime}=K\left(k^{\prime}\right)$. If $q=\exp \left(-\pi K^{\prime} / K\right)$, then one of the central theorems in the theory of elliptic functions asserts that

$$
\varphi^{2}(q)=\frac{2}{\pi} \int_{0}^{\pi / 2} \frac{d \theta}{\sqrt{1-k^{2} \sin ^{2} \theta}}=\frac{2}{\pi} K(k) .
$$

By (6), an explicit determination of $\varphi(q)$ for a certain value of $q$ also yields an explicit value for $K(k)$.

A second classical theta function is the Dedekind eta-function $\eta(z)$ defined by

$$
f(-q):=q^{-1 / 24} \eta(z):=\prod_{n=1}^{\infty}\left(1-q^{n}\right)=\sum_{k=-\infty}^{\infty}(-1)^{k} q^{k(3 k-1) / 2}, \quad q=\exp (2 \pi i z)
$$

where $|q|<1$. The exponents $k(3 k-1) / 2$ are called pentagonal numbers, and the second equality in (7) constitutes Euler's pentagonal number theorem. From (1) and (7), we easily see that

$$
G_{n}=2^{-1 / 4} q^{-1 / 24} \frac{f(q)}{f\left(-q^{2}\right)} \quad \text { and } \quad g_{n}=2^{-1 / 4} q^{-1 / 24} \frac{f(-q)}{f\left(-q^{2}\right)},
$$

when $q=\exp (-\pi \sqrt{n})$. Ramanujan likely used his values of class invariants to calculate explicitly certain products of eta-functions in both his first and lost notebooks [15], [17]. For example, he probably used the values of $G_{225}$ and $G_{25 / 9}$ to prove that

$$
e^{6 \pi / 5} \frac{f\left(-e^{-6 \pi / 5}\right)}{f\left(-e^{-30 \pi}\right)}=\frac{a+b}{a-b},
$$

where $a=(60)^{1 / 4}$ and $b=2-\sqrt{3}+\sqrt{5}$.

Ramanujan also used class invariants to determine values of the celebrated Rogers-Ramanujan continued fraction $R(q)$, defined by

$$
R(q):=\frac{q^{1 / 5}}{1}+\frac{q}{1}+\frac{q^{2}}{1}+\frac{q^{3}}{1}+\cdots, \quad|q|<1 .
$$


The behavior of $R(q)$ for $|q|=1$ is not completely understood, but if you have had a course in elementary number theory, perhaps you have shown that

$$
R(1)=\frac{\sqrt{5}-1}{2}
$$

Using the value of $G_{5}$, given in (2), we can show that

$$
R^{5}\left(e^{-2 \pi / \sqrt{5}}\right)=\sqrt{\left(\frac{5 \sqrt{5}+11}{2}\right)^{2}+1}-\frac{5 \sqrt{5}+11}{2} .
$$

To offer another example [4], Ramanujan undoubtedly used (9) to show that

$$
R\left(e^{-6 \pi}\right)=\sqrt{c^{2}+1}-c,
$$

where

$$
2 c:=1+\frac{a+b}{a-b} \sqrt{5} .
$$

Ramanujan calculated several further values of $R(q)$ in his lost notebook [16], and many of these can be found in the authors' paper [6].

In the remainder of the paper, we briefly describe some attempts and methods used to establish Ramanujan's class invariants.

In two papers [19], [20], Watson proved the 24 class invariants from Ramanujan's paper [14] that cannot be found in Ramanujan's second notebook. In the first [19], Watson devised an "empirical process" to calculate 14 of the 24 invariants, while in the second [20], he employed modular equations, which we define later in this paper, to prove 10 invariants. Watson [18] also used his empirical process to establish Ramanujan's value for $G_{1353}$. In the introduction to [19], Watson remarked, "It is intended to publish the calculations involved in the construction of the set $N+Q$ (the invariants appearing in both Ramanujan's paper [14] and the second notebook) as part of the commentary on the note-books by Dr. B. M. Wilson and myself." Although Watson and Wilson's efforts to edit Ramanujan's notebooks have been preserved in the library at Trinity College, Cambridge, Watson's calculations of these twenty-one invariants are not found there. The twenty-one values of $n$ are: $65,69,77,81,117,141,145,147,153,205,213,217,265,289,301,441,445,505$, 553,90 , and 198. Watson wrote four further papers on the calculation of class invariants, and in those he verified three additional class invariants determined by Ramanujan, namely, those for $n=81,147$, and 289. Thus, after Watson's work, and up until recent times, 18 of Ramanujan's class invariants remained to be verified.

For five of the values, $n$ is a multiple of 9, namely, $n=117,153,441,90$, and 198. The authors [7] found proofs for these values by using formulas relating $G_{9 n}$ with $G_{n}$ and $g_{9 n}$ with $g_{n}$, which we established by using one of Ramanujan's modular equations of degree 3 . All of the remaining 13 values are for $G_{n}, n=$ $65,69,77,141,145,205,213,217,265,301,445,505$, and 553. Note that each value of $n$ is the product of a small prime $(3,5$, or 7$)$ and a larger prime. Quite remarkably, the class number for each of the 13 imaginary quadratic fields $\mathbb{Q}(\sqrt{-n})$ equals 8. Moreover, there are precisely two classes per genus in each case. This is amazing! It is extremely unlikely that Ramanujan had any knowledge of imaginary 
quadratic fields, genus theory, or class numbers. However, Ramanujan must have recognized some arithmetical properties shared by these fields, although he would have expressed his ideas in a language very different from what we use today. How did Ramanujan calculate these 116 class invariants? He left no clues in his notebooks. Since Weber's methods were highly algebraic, it is very unlikely that Ramanujan journeyed along Weber's paths.

In his paper [14], Ramanujan used modular equations to calculate only a couple of simple invariants. This fact and the sentence, "The values of $G_{n}$ and $g_{2 n}$ are got from the same modular equation." [14], [16, p. 25] are the only clues to his methods that Ramanujan provided for us. It would seem that if Ramanujan had employed another type of reasoning, he would have dropped some hint about it.

Having mentioned modular equations three times already in this paper, a definition of a modular equation is overdue.

With the elliptic integral $K$ defined by (5), let $K, K^{\prime}, L$, and $L^{\prime}$ denote complete elliptic integrals of the first kind associated with the moduli $k, k^{\prime}, \ell$, and $\ell^{\prime}:=$ $\sqrt{1-\ell^{2}}$, respectively, where $0<k, \ell<1$. Suppose that

$$
n \frac{K^{\prime}}{K}=\frac{L^{\prime}}{L}
$$

for some positive integer $n$. A relation between $k$ and $\ell$ induced by (10) is called a modular equation of degree $n$. In fact, modular equations are algebraic equations. A modulus can be expressed in terms of classical theta functions. Although we suppress all details, this fact, (10), and (6) are the primary ingredients needed to show that a modular equation can alternatively be expressed as an identity relating theta functions with argument $q$ and theta functions with argument $q^{n}$.

As mentioned above, Watson [20] used modular equations to establish some of Ramanujan's invariants. We have been able to prove six of the remaining thirteen values for $G_{n}$, namely, for $n=65,69,77,141,145$, and 213 , by using modular equations of degrees $p$ and $q$, where $n=p q$, but our approach is necessarily different from that of Watson. To prove the remaining seven invariants by employing modular equations, we would need modular equations of degrees $31,41,43,53,79,89$, and 101. Apparently, only for degree 31 did Ramanujan derive a modular equation, for he recorded no modular equations for the other six degrees in his notebooks. Some of the modular equations that we employed are very complicated, and so we had to use Mathematica to effect some of our calculations. In conclusion, it seems unlikely that Ramanujan used only modular equations in these elusive computations.

In order to prove the remaining class invariants of Ramanujan, we devised two methods [7].

The first uses Kronecker's limit formula. In order to give a brief description of this formula, we need to define the Epstein zeta-function. Let $Q(u, v):=y^{-1}(u+$ $v z)(u+v \bar{z})$, where $z=x+i y$ with $y>0$. The Epstein zeta-function $\zeta_{Q}(s)$ is defined for $\sigma=\operatorname{Re} s>1$ by

$$
\zeta_{Q}(s):=\sum_{u, v}\{Q(u, v)\}^{-s}
$$

where the sum is over all pairs of integers $(u, v)$ except $(0,0)$. It is well known that $\zeta_{Q}(s)$ can be analytically continued to the entire complex $s$-plane, where $\zeta_{Q}(s)$ is analytic except for a simple pole at $s=1$. The Kronecker limit formula provides the constant term in the Laurent expansion about $s=1$. This constant term involves 
the Dedekind eta-function, which we defined in (7). The Kronecker limit formula then leads to representations for certain products of Dedekind eta-functions in terms of fundamental units. By (8), these representations allow us to calculate $G_{n}$. Our methods extend those of K. G. Ramanathan [13] who calculated some of Ramanujan's class invariants but required that $\mathbb{Q}(\sqrt{-n})$ contains only one class per genus. Zhang [22], [23] has further extended the method to give rigorous proofs of the invariants of Ramanujan that Watson [19] had "empirically" calculated.

Our second method takes Watson's ideas and employs class field theory to put the "empirical" process on a firm foundation [7]. It has been further extended by Chan to determine several new invariants [9].

It is highly doubtful that Ramanujan had any acquaintance with Kronecker's limit formula, the arithmetic of quadratic fields, or class field theory. Thus, Ramanujan's ideas still remain hidden behind an opaque curtain.

In this paper, we have made many claims without proofs (as did Ramanujan), but complete proofs or references for all our assertions can be found in [2].

\section{REFERENCES}

1. B. C. Berndt, Ramanujan's Notebooks, Part III, Springer-Verlag, New York, 1991.

2. B. C. Berndt, Ramanujan's Notebooks, Part V, Springer-Verlag, New York, 1997.

3. B. C. Berndt and H. H. Chan, Ramanujan's explicit values for the classical theta-function, Mathematika 42 (1995), 278-294.

4. B. C. Berndt and H. H. Chan, Some values for the Rogers-Ramanujan continued fraction, Canad. J. Math. 47 (1995), 897-914.

5. B. C. Berndt, H. H. Chan, and L.-C. Zhang, Ramanujan's class invariants and cubic continued fraction, Acta Arith. 73 (1995), 67-85.

6. B. C. Berndt, H. H. Chan, and L.-C. Zhang, Explicit evaluations of the Rogers-Ramanujan continued fraction, J. Reine Angew. Math. 480 (1996), 141-159.

7. B. C. Berndt, H. H. Chan, and L.-C. Zhang, Ramanujan's class invariants, Kronecker's limit formula, and modular equations, Trans. Amer. Math. Soc. (to appear).

8. B. C. Berndt and R. A. Rankin, Ramanujan: Letters and Commentary, American Mathematical Society, Providence, 1995; London Mathematical Society, London, 1995.

9. H. H. Chan, Ramanujan-Weber class invariant $G_{n}$ and Watson's empirical process, J. London Math. Soc. (to appear).

10. G. Chrystal, Algebra, 6th ed., Chelsea, New York, 1959.

11. D. A. Cox, Primes of the Form $x^{2}+n y^{2}$, Wiley, New York, 1989.

12. H. S. Hall and S. R. Knight, Higher Algebra, Macmillan, London, 1957.

13. K. G. Ramanathan, Some applications of Kronecker's limit formula, J. Indian Math. Soc. 52 (1987), 71-89.

14. S. Ramanujan, Modular equations and approximations to $\pi$, Quart. J. Math. (Oxford) 45 (1914), 350-372.

15. S. Ramanujan, Notebooks (2 volumes), Tata Institute of Fundamental Research, Bombay, 1957.

16. S. Ramanujan, Collected Papers, Chelsea, New York, 1962.

17. S. Ramanujan, The Lost Notebook and Other Unpublished Papers, Narosa, New Delhi, 1988.

18. G. N. Watson, Theorems stated by Ramanujan (XIV): a singular modulus, J. London Math. Soc. 6 (1931), 126-132.

19. G. N. Watson, Some singular moduli (I), Quart. J. Math. 3 (1932), 81-98.

20. G. N. Watson, Some singular moduli (II), Quart. J. Math. 3 (1932), 189-212.

21. H. Weber, Lehrbuch der Algebra, dritter Band, Chelsea, New York, 1961.

22. L.-C. Zhang, Kronecker's limit formula, class invariants and modular equations (II), Analytic Number Theory: Proceedings of a Conference in Honor of Heini Halberstam, vol. 2 (B. C. Berndt, H. G. Diamond, and A. J. Hildebrand, eds.), Birkhäuser, Boston, pp. 817-838. 
23. L.-C. Zhang, Kronecker's limit formula, class invariants and modular equations (III), Acta Arith. (to appear).

Department of Mathematics, University of Illinois, 1409 West Green Street, URBANA, Illinois 61801, USA

E-mail address: berndt@math.uiuc.edu

Department of Mathematics, National Chung Cheng University, Minhsiung, Chia Yi 621, TAIWAN

E-mail address: hhchan@mthmp.math.ccu.edu.tw

Department of Mathematics, Southwest Missouri State University, Springfield, MO 65804, USA

E-mail address: liz917f@cnas.smsu.edu 